\title{
Diversidad genética de aislados de Phytophthora infestans colectados en zonas productoras de papa y tomate de Guatemala
}

\author{
Genetic diversity of Phytophthora infestans isolates collected from \\ tomato and potato producing areas in Guatemala \\ José A. Ruiz-Chután, Julio E. Berdúo-Sandoval, Amilcar Sánchez-Pérez* \\ Facultad de Agronomía, Universidad de San Carlos de Guatemala, Guatemala
}

*Autor al que se dirige la correspondencia: gramisp@hotmail.com

Recibido: 16 de abril 2018 / Revisión: 12 de junio 2018 / Aceptado: 30 de julio 2018

\section{Resumen}

\begin{abstract}
Dhtophthora infestans (Mont) DeBary es el agente causal de la enfermedad conocida como tizón tardío, la cual ha sido catalogada como la enfermedad de plantas más devastadora reportada en la historia de la humanidad. Este patógeno afecta plantas de importancia económica de la familia solanaceae, como el tomate y la papa. $P$. infestans es un oomicete heterotálico y necesita de dos tipos de apareamiento, A1 y A2, para presentar reproducción sexual, la cual es la vía por la que este patógeno incrementa su grado de diversidad, a través de una recombinación de su material genético, que representa el mayor desafío para el manejo de la enfermedad. Este estudio determinó el nivel de variabilidad genética, a través del marcador molecular amplified fragment length polymorphism (AFLP), de 22 aislados de $P$. infestans colectados en diferentes zonas productoras de papa y tomate. Con el perfil de bandas generado por el marcador molecular, se realizó un análisis cluster y se elaboró un dendograma de tipo unweighted pair group method with arithmetic mean (UPGMA), con el índice de Dice, mediante una matriz de distancias genéticas. Los aislados fueron situados en tres grupos principales, los cuales responden al lugar de procedencia y al tipo de planta hospedera. Se encontró un valor de similitud de 0.49 entre los aislados analizados, por lo que se concluyó que la variabilidad genética de $P$. infestans en Guatemala es alta.
\end{abstract}

Palabras claves: amplified fragment length polymorphism (AFLP), similitud genética, unweighted pair group method with arithmetic mean (UPGMA), tizón tardío, tipo de apareamiento

\begin{abstract}
$P$ hytophthora infestans (Mont) DeBary is the causal agent of late blight disease, which has been cataloged as the most devasting plant disease in the history of humankind. This pathogen is capable of affecting economically important plants of the solanaceae family, such as tomato and potato. P. infestans is a heterothallic oomycete for which it needs two types of mating known as A1 and A2 to present a sexual reproduction, which is the main way by this pathogen increases its degree of genetic diversity through a recombination of its genetic material; this condition represents the major defiance to control this disease. This study determined the level of genetic variability, through the molecular marker amplified fragment length polymorphism (AFLP), of 22 P. infestans isolates collected in different potato and tomato producing areas in Guatemala. With the band profile generated by the molecular marker AFLP, a cluster analysis was performed creating a UPGMA dendrogram with Dice's index through a genetic distances matrix. The isolates were located in three main groups, which respond to the place of origin and the type of host plant. A similarity value of 0.49 was found among the analyzed isolates. It is concluded that genetic variability of the isolates analyzed is high.
\end{abstract}

Keywords: AFLP, genetic similarity, UPGMA, late blight, type of mating 


\section{Introducción}

En Guatemala, la enfermedad conocida como tizón tardío, es causada por el oomicete Phytophthora infestans (Mont.) DeBary, el cual tiene su origen en México, de donde se dispersó a varias regiones alrededor del mundo (Goss et al., 2014), es considerado uno de los patógenos de plantas más devastadores del mundo, prueba de ello, la hambruna irlandesa de la papa, a causa de la destrucción del cultivo en dicha región entre 1845 y 1846, lo que resultó en la muerte y desplazamiento de millones de personas. En la actualidad está catalogada entre las peores enfermedades de plantas, a pesar de las muchas investigaciones y esfuerzos realizados a través de los años (Fry, 2008).

Dicho patógeno afecta principalmente a papa y tomate, aunque también ha sido reportada en otras solanáceas que coexisten en dicho agrosistema (Forbes et al., 1998). En papa y tomate, las infecciones de tizón tardío inician como una mancha circular irregular acuosa en las hojas inferiores, así como en los brotes tiernos (Figura 1). En países en desarrollo limita gravemente la producción de estos dos cultivos de importancia económica (Erwin \& Ribeiro, 1996; Niederhauser, 1993). Bajo condiciones óptimas, $P$. infestans puede llegar a destruir el $100 \%$ del cultivo de tomate, especialmente, la producción orgánica a campo abierto e invernadero (Chowdappa et al., 2013; Stroud, Shaw, Hale, \& Steele, 2016) y entre el 50-70 \% en el cultivo de papa, produciendo pérdidas cuantificadas en USD 6.7 billones en este cultivo (Chowdappa et al., 2015; Haq, Rashid, \& Khan, 2008)

Phytophthora infestans es un patógeno hemibiotrófico y generalmente sobrevive entre cada temporada de cultivo en los tubérculos de papa, dispersándose asexualmente por vía de esporangios, los cuales pueden movilizarse por agua y viento, y así, extenderse a través de distancias más grandes (Aylor, 2003) además, es un oomicete heterotálico, lo cual significa que necesita de dos tipos de apareamientos conocidos como A1 y A2, necesarios para la reproducción sexual (Gallegly $\&$ Galindo, 1958). Cada tipo de apareamiento es bi-

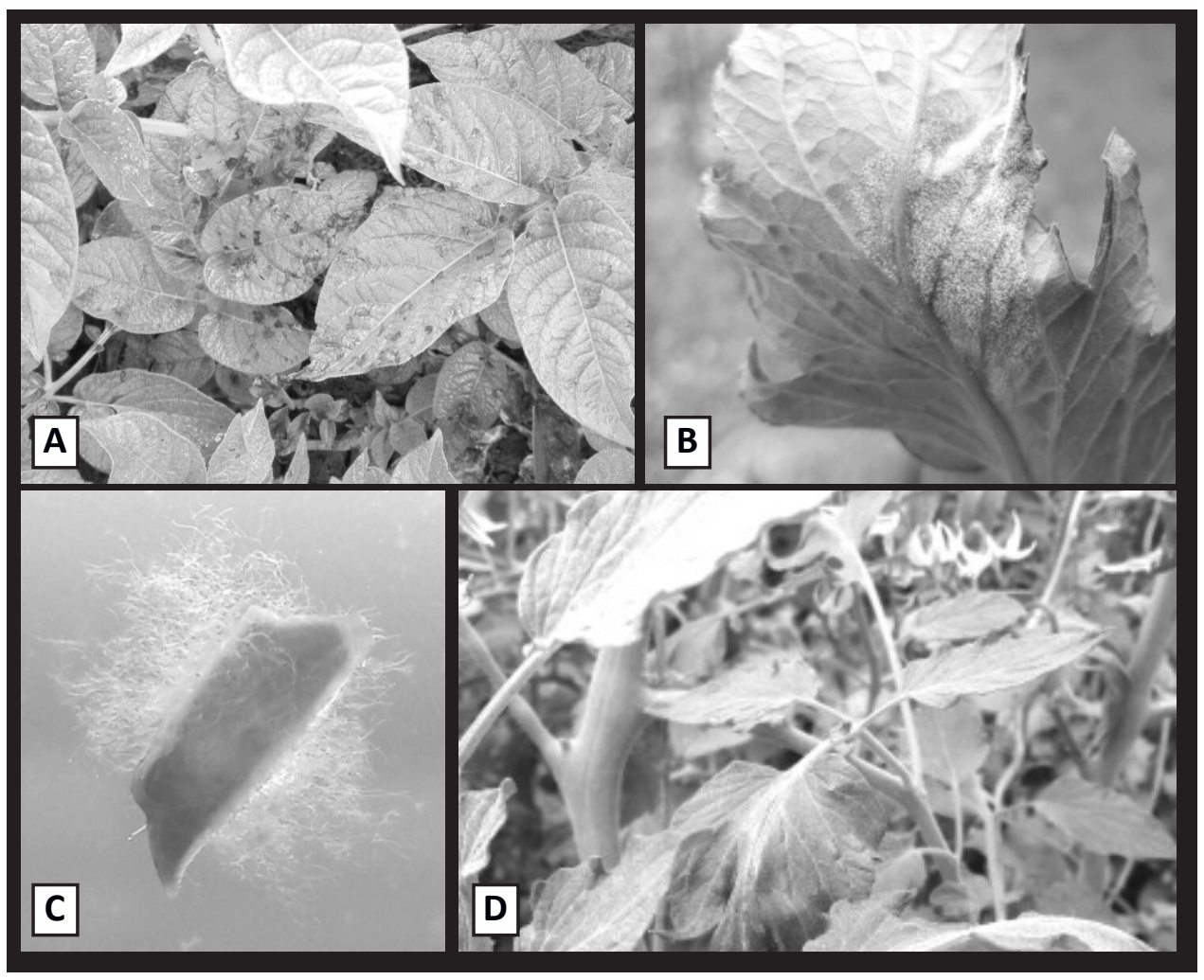

Figura 1. A, B, D Síntomas de daño por Phytophthora infestans en plantas de papa y tomate. C Aislamiento de $P$. infestans en medio de cultivo de arveja. 
sexual, capaz de producir oogonios y anteridios cuando el tipo de apareamiento opuesto está presente, cuando ocurre la fusión entre gametangios de tipos opuestos, lo cual resulta en la formación de una oospora (espora sexual). Ambos tipos de apareamiento son considerados grupos de compatibilidad y no formas sexuales, ya que el anteridio u oogonio de un tipo puede aparearse con el oogonio o anteridio del tipo opuesto, respectivamente. (Galindo \& Gallegly, 1960).

El valle de Toluca, localizado en la región central de México, es considerado como el centro de origen de P. infestans (Goodwin, Spielman, Matuszak, Bergeron, \& Fry, 1992; Gründwald et al., 2001) debido a la diversidad genética de las poblaciones del patógeno en dicha área (Flier et al., 2003; Goss et al., 2014), los tipos de apareamiento existen en una relación 1:1 (Gründwald et al., 2001), las oosporas pueden ser observadas fácilmente en plantas hospederas (Fernández-Pavía, Gründwald, Díaz-Valasis, Cadena-Hinojosa, \& Fry, 2004; Gründwald et al., 2001) y las poblaciones se reproducen sexualmente en función del equilibrio de vinculación observado entre los marcadores genéticos (Goss et al., 2014; Gründwald et al., 2001).

En otras regiones fuera de México, solo había prevalecido el tipo de apareamiento A1, que dio origen a un único genotipo clonal distribuido mundialmente y denominado US-1, cuya reproducción es típicamente asexual (Goodwin, Cohen, \& Fry, 1994). La presencia del tipo de apareamiento A2 fuera de México, fue mencionada por primera vez en Suiza en 1984 (Hohl \& Iselin, 1984). La presencia de reproducción sexual trajo como consecuencia cambios en la epidemiología del patógeno (Mayton et al., 2000) incremento de su adaptabilidad y por lo tanto, la manera en la cual la enfermedad debe ser abordada (Cooke et al., 2011; Yuen \& Andersson, 2013).

Existen claros indicios de que la diversidad genética trajo consigo cambios en las poblaciones de $P$. infestans alrededor el mundo durante la última década, los cuales concuerdan con el incremento de la severidad de los brotes de tizón tardío en los cultivos de papa y tomate a causa del aparecimiento de cepas más virulentas y resistentes a fungicidas (Chowdappa et al., 2013, 2015; Cooke et al., 2012; Fry, 2008; Li et al., 2013). Para poder realizar la identificación de genotipos individuales y explorar la diversidad genética, los marcadores moleculares son la herramienta más adecuada debido a que son neutrales a condiciones ambientales, polimórficos y efectivos en el estudio de poblaciones de patógenos (Cooke \& Lees, 2004).
El uso de marcadores moleculares ha sido ampliamente empleado alrededor del mundo en el estudio de $P$. infestans con la finalidad de conocer su diversidad y variación genética, así como el comportamiento de las poblaciones del patógeno (Alkher et al. 2015; Arafa, Elden, Olfat, Said, \& Kenta, 2018; Brurberg et al., 2011; Cárdenas et al., 2011; Kiiker, Hansen, Williams, Cooke, \& Runno-Paurson, 2018; Rekad et al., 2017; Shakya, Larsen, Cuenca, Lozoya, \& Gründwald, 2018).

Para Guatemala, el tomate (Solanum lycopersicum L.) es uno de los cultivos hortícolas de mayor importancia económica, ya que, según el informe del Ministerio de Agricultura, Ganadería y Alimentación (Maga) en el 2016, la cosecha de este cultivo se proyectó a los siete millones de quintales con un área cosechada de 11,700 manzanas. Por otra parte, el cultivo de papa (Solanum tuberosum L.) es un alimento básico y de importancia a nivel nacional, tuvo una producción de 11.8 millones de quintales con un área cosechada de 30,000 manzanas (Maga, 2016). En Guatemala, ambos cultivos ven limitada su producción debido al severo ataque de $P$. infestans que resulta en la pérdida parcial, e incluso total, de las plantaciones de dichos cultivos (Erwin \& Ribeiro, 1996; Niederhauser, 1993).

Ante esta situación, el objetivo de este estudio es conocer la variabilidad genética de $P$. infestans presente en Guatemala, debido a que los nuevos genotipos presentan resistencia a la aplicación de fungicidas y una alta adaptabilidad a diversas condiciones ambientales, lo que ha complicado el manejo de la enfermedad (Chowdappa et al., 2015; Fry, 2008) y ha repercutido negativamente en la producción de los agricultores del país. También es importante conocer la variabilidad del patógeno para realizar evaluaciones de materiales de papa y tomate que presentan resistencia genética ante el ataque de dicho patógeno, y que han sido desarrollados en centros de investigación internacionales, bajo condiciones bióticas y abióticas distintas a las presentes en Guatemala.

Para realizar dicho estudio, se tomaron muestras de tejido vegetal afectado por $P$. infestans provenientes de plantaciones de papa y tomate en Guatemala, a partir de las cuales se aisló el patógeno en medio de cultivo para realizar la extracción de ADN, a partir del micelio desarrollado. Posteriormente, se desarrolló el protocolo para el marcador molecular AFLP generando un dendrograma, a partir de la matriz de distancias genéticas generada con los datos obtenidos del gel de acrilamida. 


\section{Materiales y métodos}

\section{Colecta de tejido vegetal y aislamiento del patógeno}

Para llevar a cabo esta investigación, fueron colectadas 45 muestras de plantas de tomate y 20 de plantas de papa, de las cuales se aisló el patógeno en siete muestras de tomate y 15 de papa. Dichas muestras provinieron de los municipios de Agua Blanca, Cobán, Zunil, Ipala, Jalapa, Patzicía, Patzún, Salamá, Sanarate, San Juan Ostuncalco, San Agustín Acasaguastlán, Tajumulco, Sololá y Todos Santos Cuchumatán, como puede observarse en la Tabla 1, en la cual se detalla la ubicación precisa de cada sitio de colecta. Las muestras fueron colectadas durante los meses de mayo a septiembre, periodo donde se presentan condiciones de alta humedad (80-100 \%), temperaturas frescas $\left(18-22^{\circ} \mathrm{C}\right)$ y periodos nublados; debido a que estas condiciones potencian el desarrollo del patógeno como menciona Rick y Chetelat (1995). Cada muestra consistió de 10 hojas por cada planta afectadas por $P$. infestans en los primeros estadios de desarrollo para disminuir el riesgo de contaminación con patógenos secundarios.

Las hojas colectadas fueron trasladadas en condiciones de frío hasta el momento de realizar el aislamiento en el laboratorio de Biotecnología Vegetal de

Tabla 1

Lista de aislados de Phytophthora infestans obtenidos en diferentes regiones productoras de tomate y papa de Guatemala

\begin{tabular}{clllrl}
\hline Aislado & \multicolumn{1}{c}{ Lugar de colecta } & \multicolumn{1}{c}{ Cultivo } & \multicolumn{2}{c}{ Departamento } & \multicolumn{2}{c}{ Coordernadas } \\
\hline P1 & Agua Blanca & Tomate & Jutiapa & 14.50926 & -89.63408 \\
P2 & Cobán & Tomate & Alta Verapaz & 15.238237 & -90.252488 \\
P3 & Fuentes Georginas 1 & Papa & Quetzaltenango & 14.758588 & -91.483474 \\
P4 & Fuentes Georginas 2 & Papa & Quetzaltenango & 14.763701 & -91.483037 \\
P5 & Fuentes Georginas 3 & Papa & Quetzaltenango & 14.765284 & -91.478927 \\
P6 & Ipala & Tomate & Chiquimula & 14.535491 & -89.66710 \\
P7 & Jalapa 1 & Tomate & Jalapa & 14.537358 & -89.903819 \\
P8 & Jalapa 2 & Papa & Jalapa & 14.550435 & -90.100101 \\
P9 & Jalapa 3 & Papa & Jalapa & 14.536762 & -90.158174 \\
P10 & Patzicía 1 & Papa & Chimaltenango & 14.640814 & -90.940335 \\
P11 & Patzicía 2 & Papa & Chimaltenango & 14.645522 & -90.942162 \\
P12 & Patzicía 3 & Papa & Chimaltenango & 14.659235 & -90.945065 \\
P13 & Patzún 1 & Papa & Chimaltenango & 14.68858 & -90.99823 \\
P14 & Patzún 2 & Papa & Chimaltenango & 14.703891 & -91.012587 \\
P15 & Salamá & Tomate & Baja Verapaz & 15.077132 & -90.282946 \\
P16 & Sanarate & Tomate & El Progreso & 14.81018 & -90.213296 \\
P17 & San Juan Ostuncalco & Papa & Quetzaltenango & 14.866048 & -91.602249 \\
P18 & San Agustín Acasaguastlán & Tomate & El Progreso & 14.949777 & -89.96101 \\
P19 & San Marcos 1 & Papa & San Marcos & 15.022028 & -91.798506 \\
P20 & San Marcos 2 & Papa & San Marcos & 15.069238 & -91.835866 \\
P21 & Sololá & Papa & Sololá & 14.841253 & -91.170777 \\
P22 & Todos Santos Cuchumatán & Papa & Huehuetenango & 15.456613 & -91.501255 \\
\hline & & & &
\end{tabular}


la Facultad de Agronomía de la Universidad de San Carlos de Guatemala. Previo a la siembra se realizó un proceso de desinfección mediante inmersiones en etanol al $70 \%$, hipoclorito de sodio $1.25 \%$ y agua destilada estéril. Posteriormente, las muestras fueron sembradas en placas de Petri, en un medio de cultivo sólido de arveja suplementado con 100 ppm de penicilina y 30 ppm de procloraz. Las placas de Petri fueron incubadas en condiciones de oscuridad a $20{ }^{\circ} \mathrm{C}$. Se realizaron resiembras del hongo en el mismo medio de cultivo de arveja, sin la adición de antibiótico ni fungicidas, a intervalos de cuatro semanas para estimular el crecimiento del patógeno, debido a que, la permanencia de éste en el mismo medio de cultivo durante largos períodos, no favorece su desarrollo. Para la resiembra se empleó el medio de cultivo de arveja sin antibiótico ni fungicida.

\section{Extracción del ADN y protocolo de AFLP}

Se tomó micelio de la superficie del medio de cultivo el cual fue macerado con micropistilo agregando $400 \mu \mathrm{L}$ de amortiguador de extracción (200 mM Tris$\mathrm{HCl} ; 250 \mathrm{mM} \mathrm{NaCl} ; 25$ mM EDTA; $0.5 \%$ SDS) en un tubo de $1.5 \mathrm{~mL}$, luego los tubos se incubaron $65^{\circ} \mathrm{C}$ durante $1 \mathrm{~h}$. Posteriormente, las muestras se dejaron a temperatura ambiente y se agregó $100 \mu \mathrm{L}$ de acetato de amonio $2.0 \mathrm{M}$, colocándolas en hielo por $10 \mathrm{~min}$. Posteriormente se centrifugó a 13,000 rpm durante 4 min y la fase líquida se transfirió a un nuevo tubo al cual se le agregó $300 \mu \mathrm{L}$ de isopropanol, se centrifugó a $13.000 \mathrm{rpm}$ durante $3 \mathrm{~min}$ y se descartó la fase líquida.

La pastilla de ADN generada se lavó con etanol al $70 \%$ y se centrifugó a 13,000 rpm durante $2 \mathrm{~min}$ y se descartó la fase líquida. Tras la evaporación del etanol se suspendió la pastilla en $50 \mu \mathrm{L}$ de TE $(10 \mathrm{mM}$ Tris-HCl; 1mM EDTA) y se dejó reposar $1 \mathrm{~h}$ a temperatura ambiente. Finalmente fue almacenado a $-20^{\circ} \mathrm{C}$. La integridad y cantidad del ADN obtenido fue medida mediante electroforesis en gel de agarosa al $1.0 \%$.

El marcador molecular AFLP (Vos et al., 1995) fue utilizado debido a la alta capacidad en la identificación de regiones polimórficas en el estudio de diversidad genética de P. infestans (Abu-El Samen, Secor, \& Gudmestad, 2003; Mesa et al., 2008; Silva, Cotes, \& Marín, 2010). El análisis se realizó con el Kit AFLP Análisis System I de Invitrogen ${ }^{\circledR}$ (Zabeau \& Vos, 1993). La metodología empleada fue la siguien- te: se utilizó ADN a una concentración de $25 \mathrm{ng} / \mu \mathrm{L}$, el cual fue digerido con las enzimas de restricción EcoR1/ Msel y se incubó por $2 \mathrm{~h}$ a $37^{\circ} \mathrm{C}$, después a $70^{\circ} \mathrm{C}$ por 15 min. La ligación de los adaptadores se llevó a cabo con los reactivos del kit y se incubó durante $2 \mathrm{~h}$ a $20^{\circ} \mathrm{C}$. Seguidamente se realizó una preamplificación con la reacción en cadena de la polimerasa; el programa empleado en el termociclador fue el siguiente: $94{ }^{\circ} \mathrm{C}$ por $30 \mathrm{~s}, 56^{\circ} \mathrm{C}$ por $60 \mathrm{~s}, 72{ }^{\circ} \mathrm{C}$ por $60 \mathrm{~s}$, por 20 ciclos. Los productos amplificados se visualizaron en un gel de agarosa al $2 \%$. Se hizo una amplificación selectiva con los cebadores E-AAC + M-CTC del kit los cuales produjeron mayor polimorfismo.

Los productos de PCR se visualizaron en un gel de acrilamida al $5 \%$ para poder observar las bandas amplificadas. La tinción del gel se realizó con nitrato de plata mediante el siguiente procedimiento: inmersión en solución fijadora de ácido acético glacial $10 \%$ $\mathrm{v} / \mathrm{v}$ durante $35 \mathrm{~min}$, agua destilada durante $20 \mathrm{~min}$, solución de tinción (nitrato de plata $0.15 \% \mathrm{p} / \mathrm{v}$, formaldehído $0.15 \% \mathrm{v} / \mathrm{v}$ ) por $40 \mathrm{~min}$, agua destilada por $10 \mathrm{~s}$, solución de revelado de 5 a 6 min según la aparición de las bandas (carbonato de sodio $6 \% \mathrm{p} / \mathrm{v}$, formaldehído $0.3 \% \mathrm{v} / \mathrm{v}$, tiosulfato de sodio $5 \mathrm{ppm}$ ), solución fijadora para detener el proceso durante aproximadamente 5 min, finalmente se lavó en agua destilada. Se dejó secar por 2 días y posteriormente se realizó la lectura del perfil de las bandas, se utilizó el programa Molecular Evolutionary and Genetics Analysis MEGA 6 para observar las bandas amplificadas (Tamura, Stecher, Peterson, Filipski, \& Kumar, 2013) y Numerical Taxonomy System NTSYS (Rolfh, 1997) para realizar el análisis filogenético de los aislado de $P$. infestans obtenidos en las diferentes regiones que contempló este estudio.

\section{Análisis de datos}

Se realizó un análisis de cluster mediante la elaboración de una matriz binaria basada en la presencia (1) o ausencia (0) de cada banda en los 22 aislamientos evaluados. Esta información se utilizó para calcular una matriz de similitud con el índice de Dice, mediante el programa MEGA 6 y NTSYS, a partir de la cual, se generó un dendrograma mediante el método unweighted pair group method with arithmetic mean (UPGMA) (Sokal \& Michener, 1958) con los procedimientos Cluster y Tree. 


\section{Resultados}

Con el par de cebadores utilizados, se obtuvo 26 bandas, de las cuales el $88 \%$ fueron polimórficas. El dendrograma generado dividió los aislamientos en tres grupos principales (Figura 2). El primero de ellos incluyó cuatro aislados con un nivel de similitud de 0.54 , lo que indica un alto grado de variación. El segundo grupo incluyó 13 aislados, con un nivel de similitud de 0.66 y finalmente el tercer grupo incluyó cinco aislados, con un nivel de similitud entre ellos de 0.77.

En los resultados se observa que los aislados del patógeno fueron agrupados siguiendo un patrón con base en su lugar de procedencia, de manera que, como puede observarse en el grupo 1, los aislados provenientes de la región oriental del país, Agua Blanca (P1) y San Agustín Acasaguastlán (P18), quedaron en un mismo grupo (subgrupo 1A). Dentro de este grupo también quedaron incluidos los aislados provenientes de algunos municipios de Zunil (P4) y San Juan Ostuncalco (P17) (subgrupo 1B). El subgrupo 1A incluyó los aislados a partir de tomate, mientras que el subgru- po 1B incluyó los aislados provenientes del cultivo de papa. El grado de similitud dentro del grupo I (0.54).

Los aislados incluidos dentro del grupo 2 también pueden ser divididos en 2 subgrupos. El subgrupo 2A incluye los aislados provenientes de los municipios de Cobán (P2) y Salamá (P15) los cuales corresponden al cultivo de tomate. Este subgrupo también incluye los aislados provenientes de los municipios de Zunil (P3), Patzicía (P10) y San Marcos (P20); de donde se obtuvieron muestras de cultivo de papa. El grado de similitud dentro de este subgrupo alcanzó un valor de 0.70 . El subgrupo $2 \mathrm{~B}$ incluyó los aislados de las muestras del cultivo de papa procedentes de la región del altiplano central y región occidente del país incluyendo los municipios de Zunil (P5), Todos Santos Cuchumatán (P22), Patzicía (P11) y (P12), Sololá (P21), Patzún (P13) y (P14) y San Marcos (P19). El grado de similitud dentro de subgrupo 2B fue de 0.74 .

El grupo 3 incluye cinco aislados los cuales son divididos en dos subgrupos. El grupo 3A incluye únicamente al aislado proveniente de Ipala (P6) y muestra un coeficiente de similitud de 0.64 respecto al subgrupo

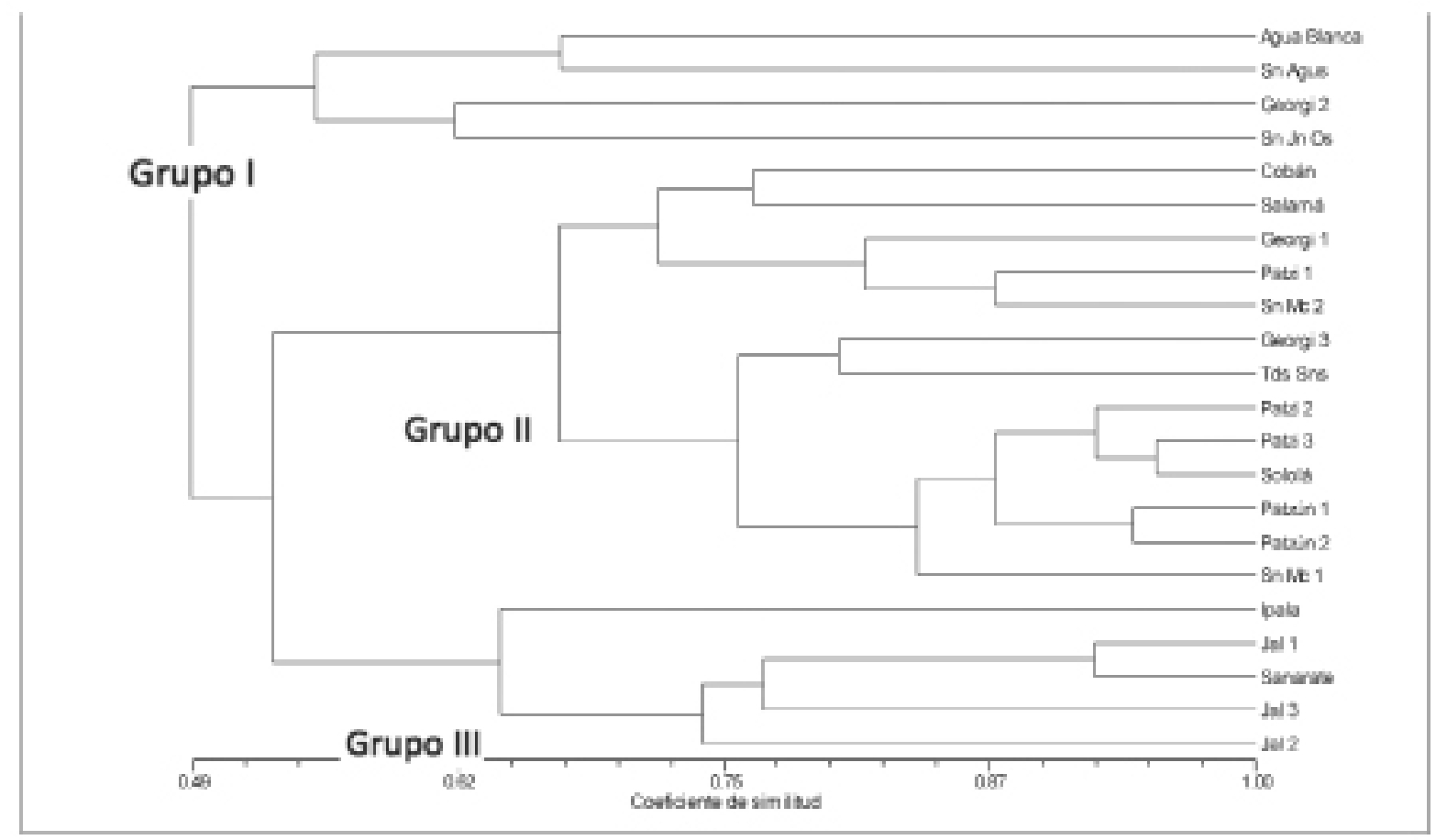

Figura 2. Dendrograma (UPGMA) generado a partir de 22 aislamientos de Phytophthora infestans mediante el marcador molecular amplified fragment length polymorphism (AFLP) que muestra la diversidad genética del patógeno en estudio en Guatemala. 
3B que incluye los aislados obtenidos de los municipios de Jalapa (P7) (P8) y (P9) y Sanarate (P16) los cuales muestran un nivel de similitud de 0.74 .

\section{Discusión}

El estudio de diversidad genética de $P$. infestans a través del marcador molecular AFLP con aislados provenientes de diferentes zonas productoras de papa y tomate para Guatemala, ha demostrado que en las zonas de estudio existe un alto grado de diversidad del patógeno. Esto puede asumirse debido a que el grado de similitud genética a través del índice de Dice mediante el método de UPGMA fue del 0.49. Al comparar dicho valor con el obtenido en el estudio de variabilidad genética de este patógeno en regiones del suroeste de Colombia (Mesa et al., 2008), mostró un nivel de similitud alrededor del 0.86 , lo que indica una mayor homogeneidad de la población, con lo cual se logra apreciar la alta variabilidad presente en el Guatemala. Situación similar presentan Silva, Jaramillo y Marín, (2009) en su estudio de variabilidad genética de dicho patógeno, afirmando nuevamente el alto grado de variabilidad presente en el territorio guatemalteco. Raigosa, Amaya, Jaramillo, Lagos y Marín (2009), obtuvieron resultados coincidentes en cuanto a la alta similitud de sus aislados (0.83).

En Costa Rica se han llegado a encontrar valores de diferenciación genética de 0.22 , donde se concluye que el flujo de genes entre poblaciones no ha sido una fuente de diversidad genética (Páez, Valverde, Gómez, $\&$ Brenes, 2004). El valor de diferenciación entre las poblaciones costarricenses se encuentra por debajo al encontrado en el presente estudio, al igual que los valores encontrados en otros estudios realizados en la región de Sur América como Ecuador, aunque en dicho país si se ha reportado la presencia de ambos tipos de apareamiento (Ordoñez et al., 2000). En el caso de Canadá, Alkher y colaboradores (2015) encontraron evidencia de recombinación genética y un incremento a la insensibilidad a mefenoxam, además de la segregación del gen Gpi, el cual según Ospina-Giraldo y Jones, 2003 es ampliamente utilizado en la caracterización de aislados de dicho patógeno por su condición multialélica, lo que ha resultado en el incremento de la diversidad de las poblaciones de $P$. infestans. Mediante el uso de microsatélites, uno de los estudios más recientes llevados a cabo en México demuestra que existe una alta diversidad genotípica, lo cual sugiere la presencia de reproducción sexual y asexual en la región central de dicho país (Shakya et al., 2018).

Dentro del subgrupo 1A los aislados incluidos responden a un lugar de procedencia cercano, así como a la misma planta hospedera, que para este caso correspondió al cultivo de tomate. Esto supone que los aislados ubicados en Agua Blanca (P1) y San Agustín Acasaguastlán (P18) han migrado en ambas vías, y por ello comparten parte de su material genético. Por otra parte, los aislados ubicados en el subgrupo 1B proceden entre ellos de una zona geográfica poco distante, razón por la cual se encuentran cercanas en cuanto a valores de distancia genética, sin embargo el grado de diversidad entre ambos subgrupos evidencia que existe una diferencia genética considerable entre ambos subgrupos (0.52)

La distribución obtenida para el caso del subgrupo $2 \mathrm{~A}$ coloca a los aislados provenientes de la región norte agrupados entre sí, siendo el caso de Cobán (P2) y Salamá P15), ambas zonas productoras de tomate y considerablemente cercanas entre sí, sin embargo, también puede observarse la presencia de aislados provenientes de los municipios de Zunil (P3), Patzicía (P10) y San Marcos (P20); aunque estos últimos disminuyen considerablemente su similitud con los aislados provenientes de Salamá (P15) y Cobán (P2). En el subgrupo 2B aparecen los aislados con procedencia de las zonas del altiplano central y zona occidental. Al indagar dentro de cada subgrupo puede observarse que la diversidad de $P$. infestans está ligada a la planta hospedera, como el caso de papa y tomate, así como el lugar de procedencia; pues el dendrograma muestra que la similitud entre los aislados del subgrupo 2B es mayor a medida que su ubicación geográfica es más cercana. La relación entre la similitud genética y los factores de tipo de plantas hospederas también fue hallada por Raigosa y colaboradores (2009), mediante la aplicación del marcador molecular RAPD en aislados de $P$. infestans.

En cuanto a la diversidad obtenida dentro del grupo 3 puede observarse nuevamente que existe relación entre el lugar de colecta y el tipo de cultivo hospedero, aunque el grado de similitud entre los aislados ubicados en este grupo es menor que la observada en el grupo 2. $\mathrm{El}$ aislado correspondiente a la zona de Sanarate (P16) muestra un nivel de similitud de 0.92 respecto al aislado obtenido de la región de Jalapa, ambas zonas productoras de tomate. Una situación similar se presenta para los otros 2 aislados de la región de Jalapa (P7) y (P8) ya que muestran un alto nivel de similitud genética, lo cual responde a su cercanía geográfica y al mismo tipo de cultivo. 
Burdon y Silk (1997) y Abu-El Samen y colaboradores (2003) establecen que la diversidad genética en la población de $P$. infestans puede estar dada por (a) el flujo de genes, (b) la recombinación sexual o asexual, y (c) por mutaciones. El presente estudio sugiere que la alta diversidad genética puede ser atribuida a la presencia de los dos tipos de apareamiento, lo cual se traduce en una reproducción sexual del patógeno incrementando de esta manera su variabilidad genética en diversas zonas del mundo, como lo demuestran los estudios de Kiiker y colaboradores (2018) y Shakya y colaboradores (2018), en los cuales la presencia de ambos tipos de apareamiento se relaciona con el incremento de la diversidad genética a causa de la reproducción sexual del patógeno.

Se sugiere que la presencia de ambos tipos de apareamiento en Guatemala puede ser debida a la cercanía geográfica con México, en donde existe una variedad de estudios que respaldan la presencia de los dos tipos de apareamiento (Gallegly \& Galindo, 1958; Goodwin et al., 1992; Goss et al., 2014; Gründwald et al., 2001; Grünwald \& Flier, 2005; Niederhauser et al., 1993; Shakya et al., 2018; Tooley, Fry, \& Villareal, 1985) situación que potencia el incremento de la variabilidad genética de las poblaciones del patógeno (Sujkowski, Goodwin, Dyer, \& Fry, 1994; van den Bosch et al., 2011). Esto, sumado a que el país es considerado como megadiverso, constituye otro factor que puede contribuir a que ambos tipos de apareamiento estén presentes en el territorio guatemalteco; además, el uso indiscriminado de fungicidas también contribuye al desarrollo de resistencia y mutaciones en el patógeno, lo cual incrementa la variabilidad genética del patógeno en el país.

Por último, es importante monitorear de forma constante la diversidad genética de $P$. infestans en el país, debido a que esta trae consigo cambios en las poblaciones y puede ser la causa de brotes severos de tizón tardío, los cuales pondrían en riesgo la producción nacional de papa y tomate a causa de la diseminación de nuevas cepas con mayor agresividad y resistencia, tanto al control químico como a los materiales desarrollados mediante mejoramiento genético. Este monitoreo podrá ayudar en el desarrollo de estrategias de control más eficientes ante futuros brotes de P. infestans en Guatemala.

\section{Agradecimientos}

Esta investigación fue cofinanciada por Digi-Usac-2017, Proyecto: 4.8.63.4.09.

\section{Referencias}

Abu-El Samen, F. M., Secor, G. A., \& Gudmestad, N. C. (2003). Genetic variation among asexual progeny of Phytophthora infestans detected with RAPD and AFLP markers. Plant Pathology, 52(3), 314325. doi:10.1046/j.1365-3059.2003.00858.x

Arafa, R. A., Elden, N., Olfat, K. S., Said, M. M., \& Kenta, M. K. (2018). Characterization of Egyptian Phytophthora infestans population using simple sequence repeat markers. Journal of General Plant Pathology, 84(2), 104-107. doi:10.1007/ s10327-018-0763-x

Alkher, H., Islam, M., Wijekoon, C., Kalischuk, L., Kawchuk, L., Peters, R.,...Daayf, F. (2015). Characterization of Phytophthora infestans populations in Canada during 2012 Epidemiology. Canadian Journal of Plant Pathology, 37(3), 305314.

Aylor, D. (2003). Spread of plant disease on a continental scale: Role of aerial dispersal of pathogens. Ecology, 84(8), 1989-1997.

Brurberg, M., Elameen, A., Le, V., Nærstad, R., Hermansen, A., Lehtinen, A., ... Yuen, J. (2011). Genetic analysis of Phytophthora infestans populations in the Nordic European countries reveals high genetic variability. Fungal Biology, 115(4-5), 335-342. doi:10.1016/j. funbio.2011.01.003

Burdon, J. J., \& Silk, J. (1997). Sources and patterns of diversity in plant pathogenic fungi. Phytopathology, 87(7), 664-9. doi:10.1094/ PHYTO.1997.87.7.664

Cárdenas, M., Grajales, A., Sierra, R., Rojas, A., González-Almario, A., Vargas, A., ... Restrepo, S. (2011). Genetic diversity of Phytophthora infestans in the Northern Andean region. BMC Genetics, 12(23). doi:10.1186/1471-2156-12-23.

Chowdappa, P., Kumar, N., Madhura, S., Kumar, M., Myers, K., Fry, W., \& Cooke, D. (2013). Emergence of 13_A2 blue lineage of Phytophthora infestans was responsible for severe out- breaks of late blight on tomato in South West India. Journal of Phytopathology, 161(1), 49-58. doi:10.1111/ jph.12031 
Chowdappa, P., Kumar, N., Madhura, S., Kumar, M., Myers, K., Fry, W., \& Cooke, D. (2015). Severe outbreaks of late blight on potato and tomato in south India caused by recent changes in the Phytophthora infestans population. Plant Pathology, 64(1), 191-199. doi:10.1111/ ppa. 12228

Cooke, D. E. L., Cano, M., Raffaele, S., Bain, R., Cooke, L., Etherington, G., ... Kamoun, S. (2012). Genome analyses of an aggressive and invasive lineage of the Irish potato famine pathogen. Plos Pathogens, 8(10), e1002940. doi:10.1371/journal. ppat. 1002940

Cooke, D. E. L., \& Lees, A. (2004). Markers, old and new, for examining Phytophthora infestans diversity. Plant Pathology, 53(6), 692-704. doi:10.1111/j.1365-3059.2004.01104.x

Cooke, D. E. L., Schepers, H., Hermansen, A., Bain, R., Dradshaw, N., Ritchie, F., ... Nielsen, B. (2011). Epidemiology and integrated control of potato late blight in Europe. Potato Research, 54, 183-222.

Erwin, D. C., \& Ribeiro, O. K. (1996). Phytophthora Diseases World Wide. St. Paul, MN: American Phytopathology Society Press.

Fernández-Pavía, S., Gründwald, N., Díaz-Valasis, M., Cadena-Hinojosa, M., \& Fry, W. (2004). Soilborne oospores of Phytophthora infestans in central Mexico survive winter fallow and infect potato plants in the field. Plant Disease, $88(1)$, 29-33. doi:10.1094/PDIS.2004.88.1.29

Flier, W., Gründwald, N., Kroon, L., Sturbaum, A., van den Bosch, T., Garay-Serrano, E., ... Turkensteen, L. (2003). The population structure of Phytophthora infestans from the Toluca Valley of central Mexico suggests genetic differentiation between populations from cultivated potato and wild Solanum spp. Phytopatology, 93(4), 382390. doi:10.1094/PHYTO.2003.93.4.382.

Forbes, G. A., Goodwin, S. B., Drenth, A., Oyarzun, P., Ordoñez, M. E., \& Fry, W.E. (1998). A global database for Phytophtora infestans. Plant Disease, 82(7), 811-818. doi:10.1094/PDIS.1998.82.7.811

Fry, W. (2008). Phytophthora infestans: The plant (and R gene) destroyer. Molecular Plant Pathology, 9(5), 385-402. doi: 10.1111/j.13643703.2007.00465.x.
Galindo, J., \& Gallegly, M. E. (1960). The nature of sexuality in Phytophthora infestans. Phytopathology, 50, 123-128.

Gallegly, M. E., \& Galindo, J. (1958). Mating type and oospores of Phytophthora infestans in nature in Mexico. Phytopathology, 48, 274-277.

Goodwin, S. B., Cohen, B. A., \& Fry, W. E. (1994). Panglobal distribution of a single clonal lineage of the Irish potato famine fungus. Proceedings of the National Academy of Science, 91(24), 1159111595.

Goodwin, S. B. Spielman, L. J., Matuszak, J. M., Bergeron, S. N., \& Fry, W. E. (1992). Clonal diversity and genetic differentiation of Phytophthora infestans populations in northern and central Mexico. Phytopatology, 82(9), 955961.

Goss, E. M., Tabima, J. F., Cooke, D. E. El, Restrepo, S., Fry, W. E., Forbes, G. A., ... Gründwald, N. J. (2014). The Irish potato famine pathogen Phytophthora infestans originated in Central Mexico rather than the Andes. Proceedings of the National Academy of Sciences, 111(24), 87918796. doi:10.1073/pnas.1401884111

Gründwald, N. J., Flier, W. G., Sturbaum, A. K., Garay-Serrano, E., van den Bosch, T. B., Smart, C. D., ... Fry, W. G. (2001). Population structure of Phytophthora infestans in the Toluca Valley region of central Mexico. Phytopatology, 91(9), 882-890. doi:10.1094/PHYTO.2001.91.9.882.

Grünwald, N. J., \& Flier, W. G. (2005). The Biology of Phytophthora infestans at Its Center of Origin. Annual Review of Phytopathology, 43, 171-190. doi: 10.1146/annurev.phyto.43.040204.135906

Haq, I., Rashid, A., \& Khan, S. (2008). Relative efficacy of various fungicides, chemicals and biochemicals against late blight of potato. Pakistan Journal of Phytopathology, 21(1), 129-133.

Hohl, H. R., \& Iselin, K. (1984). Strains of Phytophthora infestans from Switzerland with A2 mating type behaviour. Transactions of British Mycological Society, 83(3), 529-530. doi:10.1016/S00071536(84)80057-1

Kiiker, R., Hansen, M., Williams, I. H., Cooke, D. E. L., \& Runno-Paurson, E. (2018). Outcome of sexual reproduction in the Phytophthora infestans 
population in Estonian potato fields. European Journal of Plant Pathology. doi: 10.1007/ s10658-018-1483-y

Li, Y., van der Lee, T., Zhu, J., Jin, G., Lan, C., Zhu, S., ... Jacobsen, E. (2013). Population structure of Phytophthora infestans in China-Geographic clusters and presence of the EU genotype Blue 13. Plant Pathology, 62(4), 932-942.

Mesa, M. V., Mideros, M. F., Jaramillo, S., Cotes, J. M., Lagos, L. E., Pineda, R. P., ... Marín, M. (2008). Variabilidad genética de aislamientos de Phytophthora infestans procedentes del suroeste de Colombia. Revista Iberoamericana de Micología, 25(3), 167-172. doi:10.1016/S11301406(08)70039-1

Ministerio de Agricultura, Ganadería y Alimentación. (2016). El Agro en cifras, 2016. Guatemala: Dirección de Planeamiento del Ministerio de Agricultura, Ganadería y Alimentación.

Mayton, H., Smart, C. D., Moravee, B. C., Mizubuti, E. S. G., Muldoon, A. E., \& Fry, W. E. (2000). Oospore survival and pathogenicity of single oospore recombinant progeny from 23 a cross involving US-17 and US-8 genotypes of Phytophthora infestans. Plant Disease, 84(11), 1190-1196. doi:10.1094/PDIS.2000.84.11.1190

Niederhauser, J. S. (1993). Internacional cooperation in potato research and development. Annual Review of Phytopathology, 31,1-21. doi:10.1146/annurev. py.31.090193.000245

Ordoñez, M. E., Hohl, R. H., Velasco, J. A., Ramon, M. P., Oyarzun, P. J., Smart, C. D., ... Erselius, L. J. (2000). A novel population of Phytophthora, similar to P. infestans, attacks wild Solanum species in Ecuador. The American Phytopathological Society, 90(2), 197-202. doi:10.1094/PHYTO.2000.90.2.197

Ospina-Giraldo, M. D., \& Jones, R. W. (2003). Characterization of the glucose-6-phosphate isomerase gene in Phytophthora infestans reveals the presence of multiple alleles. Fungal Genetics and Biology, 40(3), 197-206. doi:10.1016/S10871845(03)00107-5

Páez, O., Valverde, R., Gómez, L., \& Brenes, A. (2004). Determinación del tipo de apareamiento con marcadores moleculares en aislamientos de Phytophthora infestans recolectados en plantaciones de papa en Costa Rica. Agronomía Costarricense, 28(2), 09-16.

Raigosa, N., Amaya, M. C. , Jaramillo, S., Lagos, L. E., \& Marín, M. (2009). Variabilidad genética de aislamientos colombianos de Phytophothora infestans (Mont) de Bary en solanáceas cultivadas en Colombia. Revista Facultad Nacional de Agronomía Medellín, 62(1), 4761-4771.

Rekad, F. Z., Cooke, D. E. L., Puglisi, I., Randall, E., Guenaoui, Y., Bouznad, Z., ... Cacciola, S. O. (2017). Characterization of Phytophthora infestans populations in northwestern Algeria during 2008-2014. Fungal Biology, 121(5), 467477. doi: 10.1016/j.funbio.2017.01.004

Rick, C., \& Chetelat, R. (1995). Utilization of related wild species for tomato improvement. Acta Horticulturae, 4(12), 21-38.

Rolfh, F. (1997). NTSYS - pc - Numerical Taxonomy and Multivariate Analysis System (Version 2.02) [Software de computación]. Exeter, New York: Applied Biostatistics.

Shakya, S. K., Larsen, M. M., Cuenca-Condoy, M. M., Lozoya-Saldaña, H., \& Gründwald, N. J. (2018). Variation in genetic diversity of Phytophthora infestans populations in Mexico from the center of origins outwards. Plant Disease, 102(8),15341540. doi: 10.1094/PDIS-11-17-1801-RE

Silva, B., Cotes, J. M., \& Marín, M. (2010) Population structure of Phytophthora infestans in potato crops from Antoquia, Boyaca, Cundinamarca, and Norte de Santander (Colombia). Agronomía Colombiana, 28(3), 375-382.

Silva, B., Jaramillo, S., \& Marín, M. (2009). Caracterización genética de aislamientos de Phytophthora infestans en las zonas productoras de papa de los departamentos de Antioquia, Boyacá, Cundinamarca y Norte de Santander (Colombia). Actualidades Biológicas, 31(90), 5-20.

Sokal, R. R., \& Michener, C. D. (1958). A statistical method for evaluating systematic relationships. University of Kansas Science Bulletin, 38, 1409-1438.

Sujkowski, L. S., Goodwin, S. B., Dyer, A. T., \& Fry, W. E. (1994). Increased genotypic diversity via 
migration and possible occurrence of sexual reproduction of Phytophthora infestans in Poland. Phytopathology, 84(2), 201- 207. doi:10.1094/ Phyto-84-201.

Stroud, J. A., Shaw, D. S., Hale, M. D., \& Steele, K. A. (2016). SSR assessment of Phytophthora infestans populations on tomato and potato in British gardens demonstrates high diversity but no evidence for host specialization. Plant Pathology, 65(2), 334-341. doi:10.1111/ppa.12407

Tamura, K., Stecher, G., Peterson, D., Filipski, A., \& Kumar, A. (2013). MEGA 6: Molecular Evolutionary Genetics Analysis version 6.0. Molecular Biology and Evolution, 30(12), 2725-2729. doi:10.1093/molbev/mst197

Tooley, P. W., Fry, W. E., \& Villareal, M. J. (1985). Isozyme characterization of sexual and asexual Phytophthora infestans populations. Journal of Heredity, 76(6), 431-435. doi:10.1093/ oxfordjournals.jhered.a110139 van Den Bosch, T., Li, Y., Evenhuis, B., Förch, M., Van Der Lee, T., \& Kessel, G. (june 2011). The population structure of Phytophthora infestans in the Netherlands during the years 2000-2009. En H. Schepers (Presidencia), Proceedings of the Thirteenth EuroBlight Workshop, St. Petersburg, Russia.

Vos, P., Hogers, R., Bleeker, M., Reijans, M., van De Lee, T., Hornes,... Zabeau, M. 1995. AFLP: A new technique for DNA fingerprinting. Nucleic Acids Research, 23, 4407- 4414.

Yuen, J., \& Andersson, B. (2013). What is the evidence for sexual reproduction of Phytophthora infestans in Europe? Plant Patology, 62(3), 485-491. doi:10.1111/j.1365-3059.2012.02685.x 